\title{
ARTICLE
}

Genetics and Genomics

\section{Integrative analysis of oncogenic fusion genes and their functional impact in colorectal cancer}

\author{
Yuri Choi ${ }^{1}$, Chae Hwa Kwon ${ }^{1}$, Seon Jin Lee ${ }^{1}$, Joonghoon Park ${ }^{2}$, Jong-Yeon Shin $^{3}$ and Do Youn Park ${ }^{1}$
}

BACKGROUND: Fusion genes are good candidates of molecular targets for cancer therapy. However, there is insufficient research on the clinical implications and functional characteristics of fusion genes in colorectal cancer (CRC).

METHODS: In this study, we analysed RNA sequencing data of CRC patients (147 tumour and 47 matched normal tissues) to identify oncogenic fusion genes and evaluated their role in CRC.

RESULTS: We validated 24 fusion genes, including novel fusions, by three algorithms and Sanger sequencing. Fusions from most patients were mutually exclusive CRC oncogenes and included tumour suppressor gene mutations. Eleven fusion genes from 13 patients (8.8\%) were determined as oncogenic fusion genes by analysing their gene expression and function. To investigate their oncogenic impact, we performed proliferation and migration assays of CRC cell lines expressing fusion genes of GTF3A-CDK8, NAGLU- IKZF3, RNF121-FOLR2, and STRN-ALK. Overexpression of these fusion genes increased cell proliferation except GTF3A-CDK8. In addition, overexpression of NAGLU-IKZF3 enhanced migration of CRC cells. We demonstrated that NAGLU-IKZF3, RNF121-FOLR2, and STRN-ALK had tumourigenic effects in CRC.

CONCLUSION: In summary, we identified and characterised oncogenic fusion genes and their function in CRC, and implicated NAGLU-IKZF3 and RNF121-FOLR2 as novel molecular targets for personalised medicine development.

British Journal of Cancer (2018) 119:230-240; https://doi.org/10.1038/s41416-018-0153-3

\section{INTRODUCTION}

Colorectal cancer (CRC) is a major cause of cancer morbidity and mortality in the world. ${ }^{1}$ Recently, promising molecularly targeted therapies have been developed, such as cetuximab (Erbitux ${ }^{\circledR}$; Merck KGaA, Germany), an epidermal growth factor receptor (EGFR)-targeted monoclonal antibody, and bevacizumab (Avas$\operatorname{tin}^{\circledR}$; Genentech Inc., USA), a vascular endothelial growth factor (VEGF)-targeted monoclonal antibody, for treatment of metastatic CRC. ${ }^{2,3}$ However, the clinical benefit of targeted therapy remains limited in CRC. Therefore, discovery and development of new molecular targets for therapy is necessary for the treatment of refractory and metastatic CRC.

Through recent advances of sequencing technology, such as next-generation sequencing (NGS), various driver mutations of colorectal cancer were identified, including adenomatous polyposis coli $(A P C)$, tumour protein p53 (TP53), SMAD family member 4 (SMAD4), phosphatidylinositol-4,5-bisphosphate 3-kinase catalytic subunit alpha (PIK3CA), KRAS proto-oncogene, GTPase (KRAS), AT-rich interaction domain 1A (ARID1A), SRY-box 9 (SOX9) and family with sequence similarity $123 \mathrm{~B}\left(\right.$ FAM123B). ${ }^{4}$ Fusion genes are good candidates of molecularly targeted therapy, such as the breakpoint cluster region protein $(B C R)$-Abelson murine leukaemia viral oncogene homologue $1(A B L)$ fusion gene in chronic myeloid leukaemia patients, and the anaplastic lymphoma kinase ( $A L K)$ fusion gene in non-small cell lung cancer. ${ }^{5,}{ }^{6}$ There are several reports of fusion genes in $C R C$, including neuron navigator 2 (NAV2)-transcription factor like 7 (TCF7L1) and R-spondin (RSPO) fusion genes. ${ }^{4}{ }^{7}$ Furthermore, Kloosterman et al. reported that various oncogenic fusions were identified in $2.5 \%$ of colon cancer, including proto-oncogene B-Raf (BRAF), neurotrophic receptor tyrosine kinase 3 (NTRK3), proto-oncogene Ret (RET), and RSPO fusion genes. ${ }^{8}$ However, the clinical implications and functional characteristics of fusion genes in CRC are unclear.

In the present study, we identified 24 fusion genes from 19 patients out of a total of 147 colon cancer patients through nextgeneration RNA sequencing (RNA-seq). To analyse the importance of these oncogenic fusion genes, we validated in vitro the functions of cyclin dependent kinase 8 (CDK8), Ikaros family zinc finger protein 3 (IKZF3), folate receptor beta (FOLR2), and $A L K$ fusion genes in CRC cell lines.

\section{MATERIALS AND METHODS}

RNA seq and analysis of fusion genes

Paired-end RNA seq was performed in our previous study. ${ }^{9}$ Briefly, fresh frozen tissue samples were collected from patients who had resection of the primary tumour at the Pusan National University Hospital and Chonnam National University Hwasun Hospital from 2008 to 2012. In total, 147 tumour samples, including 47 matched normal samples, were analysed. Tumour samples with at least $60 \%$

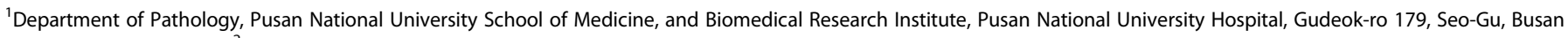

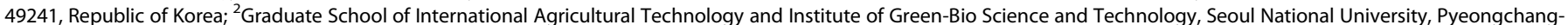
gun, Gangwon-do 232-916, Republic of Korea and ${ }^{3}$ Genomic Medicine Institute (GMI), Medical Research Center, Seoul National University, Seoul 159-781, Republic of Korea Correspondence: Do Youn. Park (pdy220@pusan.ac.kr) 
tumour cells without significant mucin or inflammatory cell contaminations examined by mirror image histological analysis were used in the present study. After total RNA was isolated by using RNAiso Plus (Takara, Japan), RNA-seq libraries were generated by TruSeq RNA Sample Preparation Kit according to the manufacturer's instructions (Illumina, U.S.). Sequencing data were aligned to the National Center for Biotechnology Information (NCBI) human reference genome (hg19) and also aligned to a custom human reference CDNA. ${ }^{10}$

Fusion genes were filtered by GFP algorithm. We determined 101 in-frame shift fusion genes that were not expressed in normal tissues. We applied additional criteria, such as spanning reads $\geq 10$ and chromosomal distance $\geq 100 \mathrm{~kb}$, to identify intrachromosomal rearrangements. Cross-validation was performed using deFuse ${ }^{11}$ and FusionMap. ${ }^{12}$

Non-synonymous somatic mutation analysis

We repeated non-synonymous somatic mutation analysis on 19 CRC patients positive for fusion genes. Single nucleotide variants (SNVs) were determined based on Fisher Strand values $>30.0$ and Qual By Depth values <2.0 using Genome Analysis Toolkit (GATK, version 2015.1-3.4.0-1-ga5ca3fc). SNVs were filtered according to the following criteria: (1) read depth at position $\geq 10$, (2) alteration read depth $\geq 2$, (3) allele ratio at position $\geq 3 \%$, (4) region in exon, (5) type of change: frameshift, non-synonymous, stop-gain, or stop-loss. To remove potential germline variants, we used dbSNP137 at minor allele frequency $>1 \%$ of samples $^{13}$ and variants from four matched normal tissue samples.

\section{Reverse transcription-polymerase chain reaction and Sanger} sequencing

Fusion genes were validated by reverse transcription-polymerase chain reaction (RT-PCR) with primers detecting the fusion gene break sites. Total RNA was extracted from fresh frozen tissues and CRC cell lines by using RNAiso Plus (Takara, Japan). RNA samples (500 ng) were reverse-transcribed into cDNA using M-MLV Reverse Transcriptase (Promega, U.S.). PCR reactions were conducted for 4 min at $94^{\circ} \mathrm{C}$, and 35 cycles for $40 \mathrm{~s}$ at $94^{\circ} \mathrm{C}, 40 \mathrm{~s}$ at melting temperature (TM) according to each primer set, $40 \mathrm{~s}$ at $72^{\circ} \mathrm{C}$, and $7 \mathrm{~min}$ at $72^{\circ} \mathrm{C}$. Detailed information of primers is listed in Table S1. PCR products after PCR purification (Cosmogenetech, Korea) were confirmed by Sanger sequencing. To validate the transfection status of vectors transfected into CRC cell lines, RT-PCR was performed according to the same method as described above (Tables S1 and S2).

Quantitative RT-PCR was performed by using SYBRGreen (Applied Biosystems, Life Technologies, U.S.) and the fluorescence reader, Corbett Rotor-Gene 6000 (Qiagen Inc., U.S.). Data were normalised to glyceraldehyde 3-phosphate dehydrogenase (GAPDH), and mRNA abundance was calculated using the $2^{-\triangle \Delta C T}$ method.

\section{Construction of overexpression vector for fusion genes}

A short variant of two RNF121-FOLR2 isoforms was used in the experiment, because the long variant contained an untranslated region in the middle of the transcription sequences. After they were amplified from the patient harbouring the RNF121-FOLR2 fusion gene, FOLR2 and RNF121-FOLR2 sequences were ligated into pcDNA3.1/V5-His B vectors (Invitrogen, U.S.) via one-step ligation method. ${ }^{14}$ For the other fusion genes, donor and acceptor gene overexpression vectors ligated in pCMV6-Entry were purchased from Origene, U.S., and then fusion gene vectors were constructed by combining the target regions of the donor and acceptor gene vectors based on pCMV6-Entry (Cosmogenetech, Korea). All vectors were confirmed by Sanger sequencing.

Cell proliferation and migration assays

CRC cell lines, DLD-1 and SW620, were obtained from the Korean Cell Line Bank (Korea) and maintained in RPMI-1640 (Gibco, U.S.), supplemented with $10 \%$ foetal bovine serum (Gibco, U.S.), at $37^{\circ} \mathrm{C}$ in a $5 \% \mathrm{CO}_{2}$ incubator. Cells $\left(1.5 \times 10^{4}\right)$ were seeded in triplicate in 24-well plates. After $24 \mathrm{~h}$, adhered cells were transfected with empty, acceptor gene, or fusion gene vector for 48 and $72 \mathrm{~h}$. To check cell viability, 3-(4,5-dimethylthiazol-2-yl)-2,5-diphenyltetrazolium bromide (MTT; Sigma-Aldrich, U.S.) was added to washed cells at a final concentration of $0.5 \mathrm{mg} / \mathrm{mL}$. After 1-h incubation, absorbance was measured by spectrophotometry at $570 \mathrm{~nm}$ (Hewlett packard, U.S.).

After 48-h transfection of CRC cells with the appropriate vectors, migration assay was performed in triplicate using membrane filters ( $8 \mu \mathrm{m}$ pore size) in disposable 96 -well chemotaxis chambers (Neuro Probe; Gaithersburg, U.S.). Cells $\left(3 \times 10^{3}\right.$ and $5 \times 10^{3}$ of DLD-1 and SW620, respectively) were resuspended (50 $\mu \mathrm{L})$, loaded into the upper chambers on membrane filters coated with $5 \mathrm{mg} /$ $\mathrm{mL}$ fibronectin, and incubated for $4 \mathrm{~h}$ at room temperature. After $18 \mathrm{~h}$, cells beneath the membrane were fixed, stained with Hoechst33342 (Sigma-Aldrich, U.S.), and counted by fluorescence microscopy at $10 \times$ magnification.

\section{Statistical analysis}

Clinicopathological information was last updated in October 2017, more than 9 years after the first patient's diagnosis. Clinicopathological features, including sex, tumour location, histological differentiation, perineural invasion, invasion depth, lymphovascular invasion, lymph node metastasis, and status of microsatellite instability, were analysed for the presence of fusion genes using the Student's $t$-test, $X^{2}$ test, or Fisher's exact test. Cumulative survival plots were generated using the Kaplan-Meier method, and significance was compared using the log-rank test. Statistical significance was set at $P<0.05$. Statistical calculations were performed using IBM SSPS version 23.

\section{RESULTS}

Identification and validation of fusion genes in CRCs

We performed RNA-seq of 147 CRC patients (147 tumour tissues and 47 matched normal tissues) in our previous study. ${ }^{9}$ We applied a screening process to identify significant fusion genes in CRC (Fig. 1a). Using GFP algorithm, we identified 2460 fusion genes, including 101 in-frame shifts that were not expressed in normal tissues. Next, we filtered for additional criteria, such as spanning reads $\geq 10$ and intrachromosomal rearrangement distance cutoff $\geq 100 \mathrm{~kb}$, and identified 25 fusion genes. FusionMap and deFuse algorithms were applied to the 25 genes for cross-validation, and all genes corresponded to two out of three algorithms (Fig. 1b). We validated 24 of 25 fusion genes via RT-PCR and Sanger sequencing (12.9\%) (Fig. 1C, Table 1). We reported six of these fusion genes, including tropomyosin 3 (TPM3)-NTRK1, lamin $\mathrm{A} / \mathrm{C}(L M N A)-N T R K 1$, protein tyrosine phosphatase receptor type K (PTPRK)-RSPO3, N-acetyl-alpha-glucosaminidase (NAGLU)IKZF3, general transcription factor IIIA (GTF3A)-CDK8, and RAS p21 protein activator 1 (RASA1)-LOC644100, in our previous study. ${ }^{9}$ Striatin (STRN)-ALK fusion was previously reported in lung cancer, thyroid carcinomas, and colon adenocarcinoma. ${ }^{15-17}$ Additionally, we found $A P C$ and NDRG family member 3 (NDRG3) fusions consisting with unreported partner genes. ${ }^{18,19}$ Most of the fusion genes we identified were from different individual patients $(n=1)$, except NTRK1 $(n=3)$ and RSPO3 fusions $(n=2)$ (Table 1).

Clinicopathological significance of CRCs positive for fusion genes The clinicopathological features was investigated based on CRC patients positive $(n=19)$ for the 24 fusion genes. Microsatellite instability (MSI-H) associated with the presence of fusion genes $(P$ $=0.025$ ) and CRC positive for fusion genes exhibited smaller size and poor histological differentiation compared to CRC negative for fusion genes $(P=0.050$ and $P=0.093$, respectively). Other clinicopathological features, including sex, tumour location, 
a

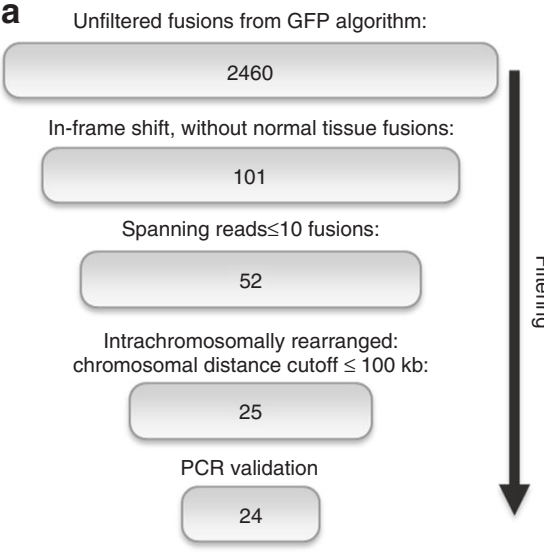

b

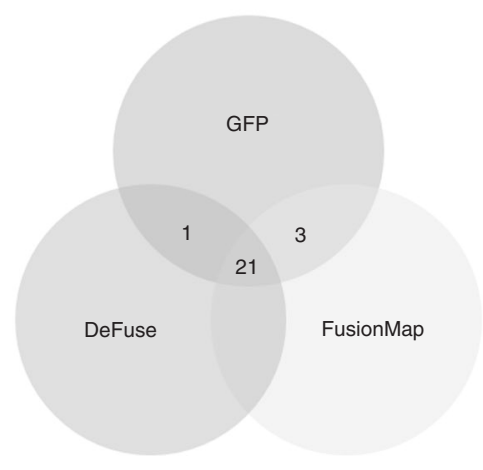

C

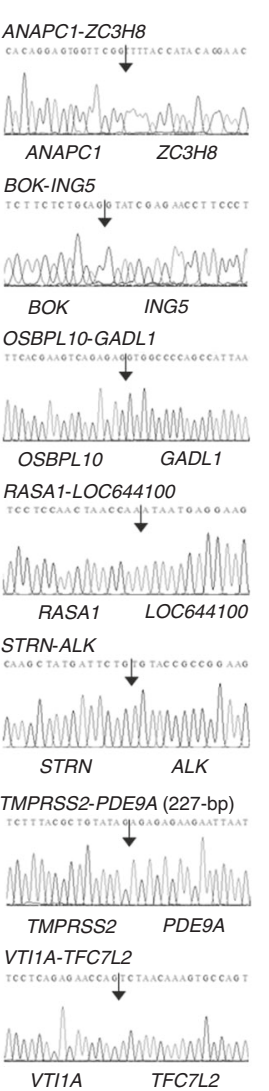

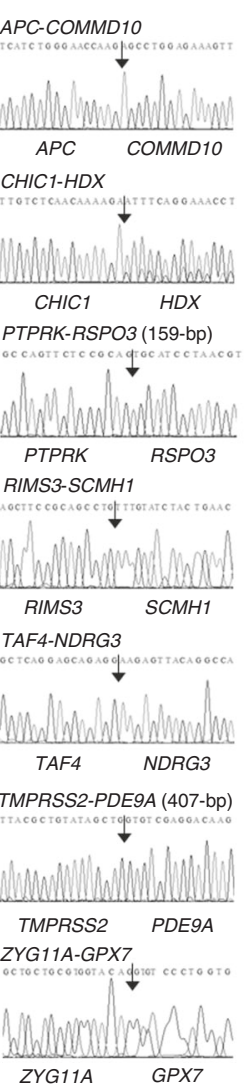
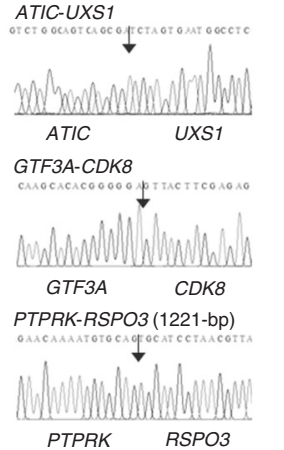

RNF121-FOLR2 (249-bp)
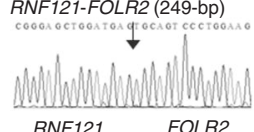

TMOD3-MAPK6 (167-bp)
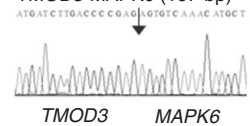

TRIM24-BRAF

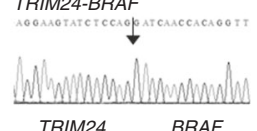

TRIM24 BRAF

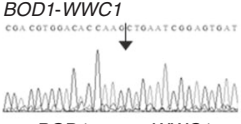

BOD1 WWC1

NAGLU-IKZF3

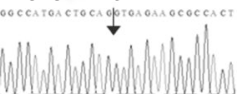

NAGLU IKZF3

RAB1B-DPP3

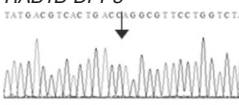

RAB1B DPP3

RNF121-FOLR2 (423-bp)

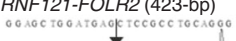

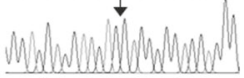

RNF121 FOLR2

TMOD3-MAPK6 (282-bp)

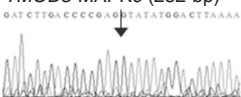

TMOD3 MAPK6

USP32-NSF

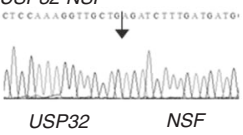

Fig. 1 Identification and validation of fusion genes in CRC. a Overview of fusion gene filtering process. $\mathbf{b}$ Cross-validation with deFuse and FusionMap algorithms prior to PCR validation. GFP algorithm was used as the first detection tool, and then deFuse and FusionMap algorithms were applied to the 25 genes. c RT-PCR validation. Sanger sequencing confirmed 24 out of 25 fusion genes. An arrow denotes a junction point of a fusion gene

perineural invasion, invasion depth, lymphovascular invasion, and lymph node metastasis, were not associated with fusion gene status of CRC samples (Table 2). CRC patients positive for fusion genes were diagnosed at diverse clinical stages (stage $\mathrm{I}=3$, stage $\|=5$, stage $I I I=11$ ). Kaplan-Meier survival analysis showed no significant correlation between overall survival and presence of fusion genes in 147 CRC patients (fusion negative-CRC $=90.4 \pm$ 2.70 months; fusion positive-CRC $=88.4 \pm 4.46$ months, $P=0.277$ ) (Fig. S1).

Mutually exclusive oncogenicity of fusion genes in colorectal cancers

SNVs were analysed compared to the Cancer Genome Atlas (TCGA) data. ${ }^{4}$ To reduce the potential error of germline variants in the RNA-seq analysis, we used paired tumour-normal tissue data and dbSNP137. Fusion genes identified in this study were mutually exclusive to oncogenic mutations (Fig. 2). Somatic mutations in various tumour suppressor genes were observed in CRC patients positive for fusion genes. In contrast, in many cases, patients harbouring these fusion genes did not contain non-synonymous somatic mutations in KRAS, NRAS proto-oncogene, GTPase (NRAS), $P I K 3 C A, B R A F$, or other putative oncogenes. In addition, there were no somatic mutations in either partner genes within fusions. It was noted that one patient contained non-synonymous somatic mutations in both tumour suppressor genes and oncogenes in addition to tropomodulin 3 (TMOD3)-mitogen-activated protein kinase 6 (MAPK6) fusion gene, despite being microsatellite stable. Interestingly, it had three mutations in the DNA polymerase epsilon catalytic subunit (POLE) gene (NM_006231: exon39: c.5239G >A: p. D1747N, exon35: c.4522C>T: p.R1508C, and exon14: c.1376C>T: p.
S459F), which was previously reported as a driver of hypermutated $\mathrm{CRC}^{4}$, while the other patients did not have a POLE mutation or had only one POLE mutation.

Prediction of oncogenic fusion genes in colorectal cancers Among 24 fusion genes, 11 fusions (APC-COMM domain containing 10 (COMMD10), GTF3A-CDK8, LMNA-NTRK1, NAGLU-IKZF3, PTPRK-RSOP3， RASA1-LOC644100， RNF121-FOLR2， STRN-ALK, TMOD3-MAPK6, TPM3-NTRK1, and tripartite motif containing 24 (TRIM24)-BRAF) from 13 patients (8.8\%) were identified as oncogenic fusion genes according to their expression value, determined from outlier analysis, and function as an oncogene, tumour suppressor gene, or kinase (Fig. 3, Table 1). Expression analysis revealed that patients harbouring the fusion genes, GTF3A-CDK8, LMNA-NTRK1, NAGLU-IKZF3, PTPRK-RSOP3, RASA1LOC644100, RNF121-FOLR2, STRN-ALK, or TPM3-NTRK1, exhibited higher expression of donor or acceptor gene compared to the other patients (Fig. 3a). Furthermore, the acceptor genes of GTF3ACDK8, LMNA-NTRK1, PTPRK-RSOP3, STRN-ALK, TMOD3-MAPK6, TPM3-NTRK1, and TRIM24-BRAF were characterised to have kinase functional domains and oncogenicity (Fig. 3b). Functional domain analysis of APC-COMMD10 and RASA1-LOC644100 fusion genes showed truncation of APC (293 aa/325 aa) and RASA1 (179 aa/253 aa), respectively, which may induce loss of function of each tumour suppressor (Fig. S2).

Functional validation of CDK8, IKZF3, FOLR2, and ALK fusion genes in colorectal cancers

We selected GTF3A-CDK8, LMNA-NTRK1, NAGLU-IKZF3, RNF121FOLR2, STRN-ALK, and TPM3-NTRK1 for functional analysis, 


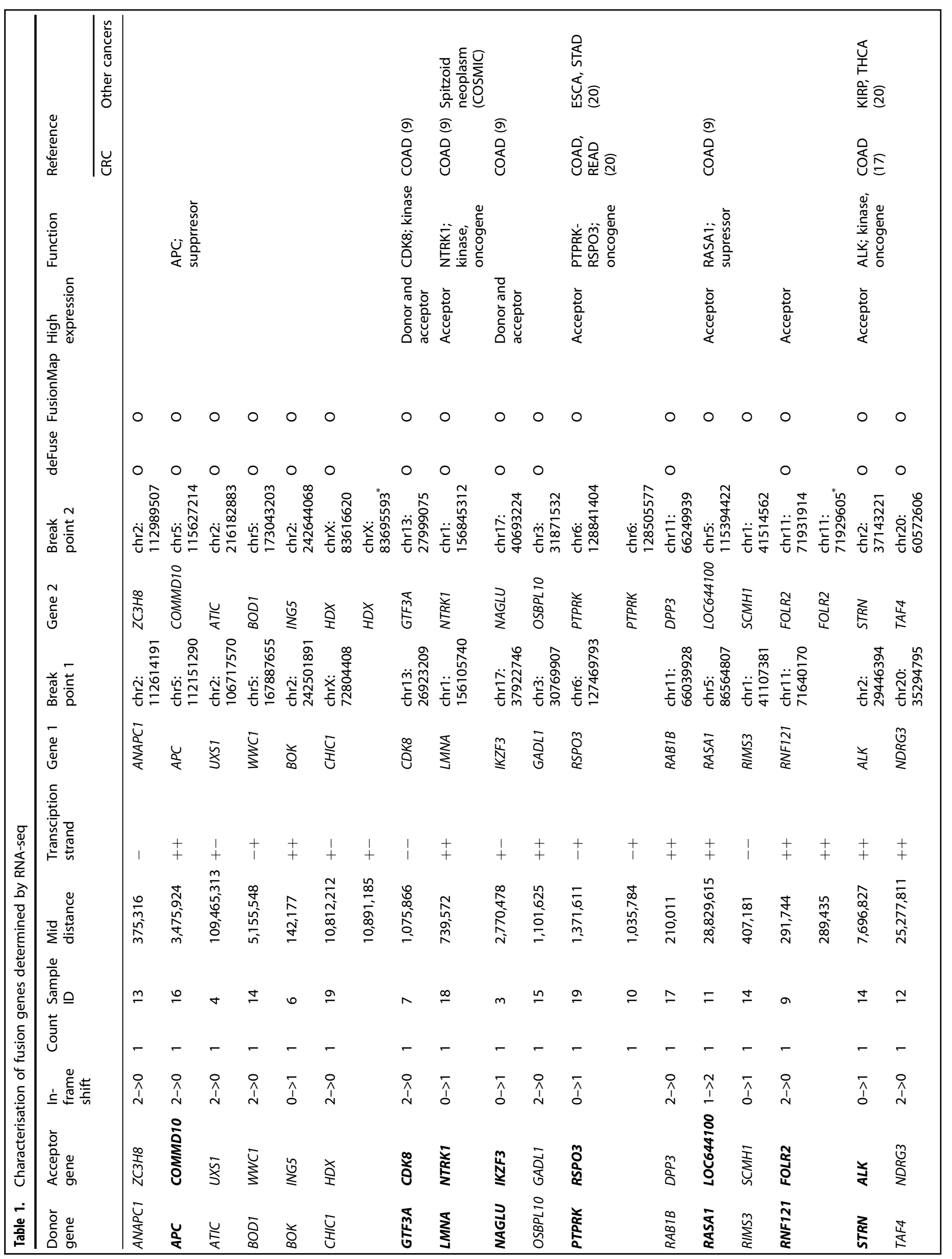




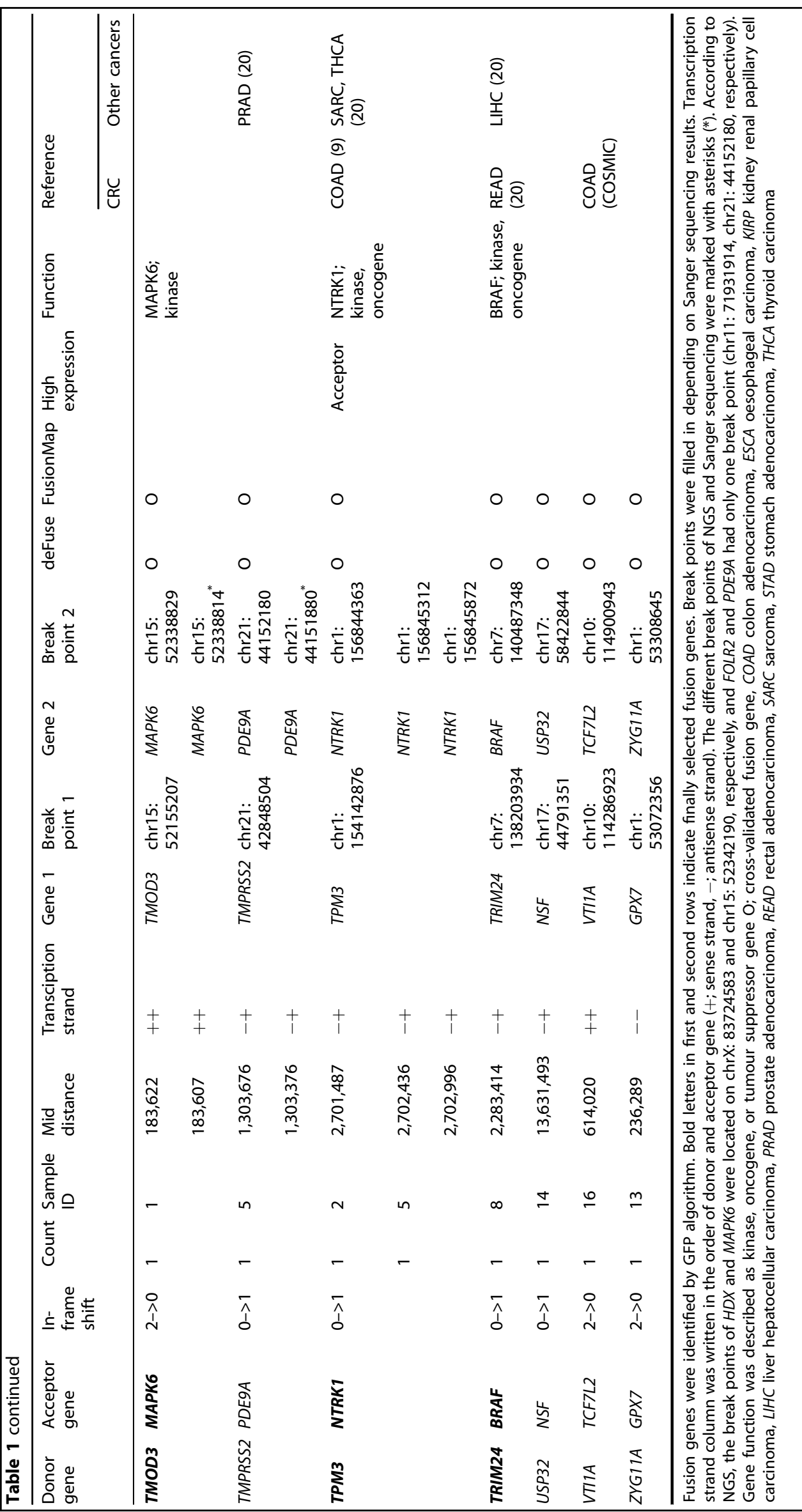


Table 2. Relationship between fusion genes revealed by RNA-seq and clinicopathological characteristics in 147 patients with colorectal cancer

\begin{tabular}{|c|c|c|c|c|}
\hline & \multirow[t]{2}{*}{ (No.) } & \multicolumn{2}{|c|}{ Fusion genes } & \multirow[t]{2}{*}{$P$ value } \\
\hline & & Absent & Present & \\
\hline Age (years) & 147 & $60.1 \pm 1.00$ & $60.7 \pm 2.73$ & 0.852 \\
\hline Size $(\mathrm{cm})$ & 147 & $5.81 \pm 0.19$ & $4.76 \pm 0.33$ & 0.050 \\
\hline Sex & & & & 0.819 \\
\hline Male & 77 & $68(88.3)$ & 9 (11.7) & \\
\hline Female & 70 & $60(85.7)$ & $10(14.3)$ & \\
\hline Location & & & & 0.162 \\
\hline Right colon & 56 & $46(82.1)$ & $10(17.9)$ & \\
\hline Left colon & 91 & $82(90.1)$ & $9(9.9)$ & \\
\hline Histological type ${ }^{a}$ & & & & 0.093 \\
\hline Well & 27 & $23(85.2)$ & $4(14.8)$ & \\
\hline Moderately & 111 & $99(89.2)$ & $12(10.8)$ & \\
\hline Poorly + Mucinous & 9 & $6(66.7)$ & $3(33.3)$ & \\
\hline Invasion depth ${ }^{\mathrm{b}}$ & & & & 0.463 \\
\hline $\mathrm{T} 1$ & 1 & $1(100.0)$ & $0(0.0)$ & \\
\hline $\mathrm{T} 2$ & 16 & $13(81.3)$ & $3(18.8)$ & \\
\hline T3 & 108 & $95(88.0)$ & $13(12.0)$ & \\
\hline $\mathrm{T} 4$ & 22 & $19(86.4)$ & $3(13.6)$ & \\
\hline Perineural invasion & & & & 0.452 \\
\hline Negative & 89 & $76(85.4)$ & $13(14.6)$ & \\
\hline Positive & 58 & $52(89.7)$ & $6(10.3)$ & \\
\hline Lymphovascular emboli & & & & 0.553 \\
\hline Negative & 116 & $102(87.9)$ & $14(12.1)$ & \\
\hline Positive & 31 & $26(83.9)$ & $5(16.1)$ & \\
\hline \multicolumn{5}{|l|}{ Lymph node } \\
\hline 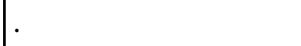 & & & & 0.521 \\
\hline Negative & 72 & $64(88.9)$ & $8(11.1)$ & \\
\hline Positive & 75 & $64(85.3)$ & $11(14.7)$ & \\
\hline Microsatellite status & & & & 0.025 \\
\hline MSS+MSI-L & 126 & $113(89.7)$ & $13(10.3)$ & \\
\hline MSI-H & 21 & $15(71.4)$ & $6(28.6)$ & \\
\hline
\end{tabular}

according to their expression values. However, we recently investigated the functional roles of LMNA-NTRK1 and TPM3NTRK1 in $\mathrm{CRC}^{9}$ and the oncogenic role of PTPRK-RSOP3 was previously reported. ${ }^{7}$ Therefore, we performed in vitro functional analysis of only four fusion genes, GTF3A-CDK8, NAGLU-IKZF3, RNF121-FOLR2, and STRN-ALK, in the present study. First, RT-PCR was conducted to detect the presence of the fusion genes in various CRC cell lines, including DLD-1, HT-29, SW480, SW620, and HCT15. The short variant of RNF121-FOLR2 fusion gene was confirmed present in HT-29 cells, whereas no other fusion genes were detected in the cell lines (Fig. S3A). To accurately validate this, the full sequence of RNF121-FOLR2 was amplified in HT-29 cells and the sequencing result aligned with reference sequences (Fig. S3B). However, RNF121-FOLR2 expression in HT-29 cells was significantly lower than that in the CRC patient, which was not suitable for knockdown experiments (Fig. S3C).

Next, we performed MTT assay to determine the effect of the fusion genes on cell proliferation (Fig. 4a). No significant change was observed in cells overexpressing GTF3A-CDK8. Cell proliferation of CRC cell lines overexpressing NAGLU-IKZF3 and RNF121-
FOLR2 increased at both 48 and $72 \mathrm{~h}$ after transfection, compared to the negative control. In addition, CRC cells overexpressing $S T R N-A L K$ grew rapidly at $72 \mathrm{~h}$, compared to the negative control. To investigate the effect of the fusion genes on migration capacity of CRC cells, we performed migration assay $48 \mathrm{~h}$ after transfection of overexpression vectors. CRC cells overexpressing NAGLU-IKZF3 exhibited increased migration capacity, compared to cells transfected with empty or IKZF3 vector (Fig. 4b, c). Especially, migration of SW620 cells overexpressing NAGLU-IKZF3 increased by 3.5 -fold (Fig. 4b, c). However, CRC cells transfected with GTF3ACDK8, RNF121-FOLR2, or STRN-ALK did not show aberrant migration (data not shown). Additionally, we conducted cell cycle analysis via propidium iodide staining, but no apoptotic effect was observed by the fusion genes (Fig. S4).

Therefore, our results demonstrated that expression of NAGLUIKZF3, RNF121-FOLR2, and STRN-ALK enhanced cell proliferation, while expression of NAGLU-IKZF3 promoted cell migration.

\section{DISCUSSION}

We identified 24 fusion genes, including novel fusion genes that were not previously reported, through RNA-seq of 147 CRC patients. Among those, we determined that 11 fusion genes were oncogenic according to gene expression and function. Finally, we validated in vitro the oncogenic functions of CDK8, IKZF3, FOLR2, and $A L K$ fusion genes in CRC cell lines.

Several studies identified various fusion genes in $C R C$, including NAV2-TCF7L1, RSPO, BRAF, NTRK3, RET, and RAS fusion genes, as good candidates of molecularly targeted therapy. ${ }^{4}$, 7, 8 Our strict filtering approach uncovered novel fusion genes in CRC, compared to fusion gene databases, such as Tumour Fusion Gene Data Portal ${ }^{20}$ and Catalogue of Somatic Mutations in Cancer (COSMIC), and other studies. We reported 14 novel fusions out of the 24 fusion genes identified, including anaphase promoting complex subunit 1 ( $A N A P C 1)$-zinc finger $\mathrm{CCCH}$-type containing 8 (ZC3H8), $A P C-C O M M D 10$, 5-aminoimidazole-4-carboxamide ribonucleotide formyltransferase (ATIC)-UDP-glucuronate decarboxylase 1 (UXS1), biorientation of chromosomes in cell division 1 (BOD1)-WW and C2 domain containing 1 (WWC1), $\mathrm{Bcl} 2$ family apoptosis regulator (BOK)-inhibitor of growth family member 5 (ING5), cysteine rich hydrophobic domain 1 (CHIC1)-highly divergent homeobox (HDX), oxysterol binding protein like 10 (OSBPL10)-glutamate decarboxylase like 1 (GADL1), Ras-related protein Rab-1B (RAB1B)-dipeptidyl peptidase $3(D P P 3)$, regulating synaptic membrane exocytosis 3 (RIMS3)-Scm polycomb group protein homologue 1 (SCMH1), RNF121-FOLR2, TATA-box binding protein associated factor 4 (TAF4)-NDRG3, TMOD3-MAPK6, ubiquitin specific peptidase 32 (USP32)-N-ethylmaleimide sensitive factor (NSF), and zyg-11 family member A (ZYG11A)-glutathione peroxidase 7 (GPX7). MAPK6$T M O D 3$, with different direction and junction point from TMOD3MAPK6, was identified by Hu et al. ${ }^{20}$ In our study, we confirmed expression of RNF121-FOLR2 in HT-29 cells, which was contrary to Nome et al. who identified expression of fusion genes via RNA-seq of various CRC cell lines, including HT-29. ${ }^{21}$ The discrepancy may be due to the low expression of RNF121-FORL2 in HT-29 cells, which may result in significantly fewer mapping reads by RNA-seq. RNF121-FOLR2 expression in the CRC patient was 10 times more abundant than in HT-29 cells (Fig. S3C).

Clinicopathological analysis determined that MSI status, more specifically MSI-H, of CRC patients positive for fusion genes correlated with the presence of fusion genes. The association of the presence of fusion genes and MSI status is unclear; fusion genes are suggested to be upregulated in MSI tumours. Kloosterman et al. reported that gene fusion tended to occur in CRC patients with MSI-H $(P=0.007),{ }^{8}$ and Kalvala et al. reported that $55 \%$ of CRC patients with MSI tended to have fusion genes $(n=$ 54; $P=0.166){ }^{22}$ Continuous studies are required to clarify the relation between the presence of fusion genes and MSI. Histotype- 


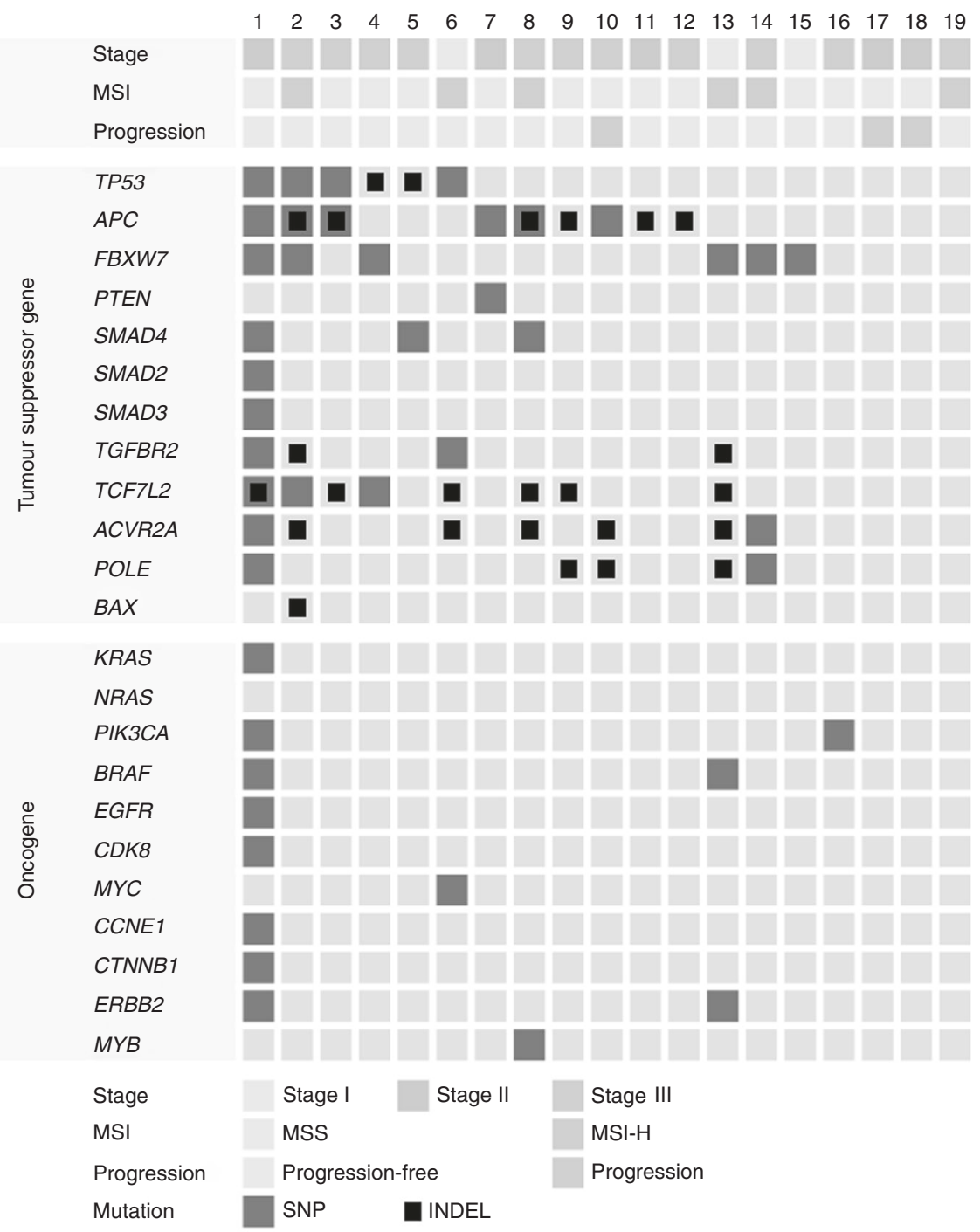

Fig. 2 Schematic overview of mutation profiling. Mutual exclusivity of fusion genes with oncogenic mutations was represented, except TMOD3-MAPK6. The numbers on top designate each patient sample ID with fusion genes; 1, TMOD3-MAPK6; 2, TPM3-NTRK1; 3, NAGLU-IKZF3; 4, ATIC-UXS1; 5, TMPRSS2-PDE9A and TPM3-NTRK1; 6, BOK-ING5; 7, GTF3A-CDK8; 8, TRIM24-BRAF; 9, RNF121-FOLR2; 10, PTPRK-RSPO3; 11, RASA1LOC644100; 12, TAF4-NDRG3; 13, ANAPC1-ZC3H8 and ZYG11A-GPX7; 14, BOD1-WWC1, RIMS3-SCMH1, STRN-ALK, and USP32-NSF; 15, OSBPL10GADL1; 16, APC-COMMD10 and VTI1A-TCF7L2; 17, RAB1B-DPP3; 18, LMNA-NTRK1; 19, CHIC1-HDX and PTPRK-RSPO3

genotype associations based on fusion genes have been extensively investigated and some reports suggest that harbouring a fusion gene was significantly associated with certain histological types of cancer. ${ }^{23-26}$ In the present study, correlation analysis regarding histological types revealed that CRC patients harbouring fusion genes tended to display poor histological differentiation compared to CRC negative for fusion genes ( $P=$ 0.093). However, more cases are necessary to be reported for statistical relevance, since this result only reveals trends.

We implicated the oncogenic potential of these fusion genes because our SNV profiling determined that the presence of fusion genes was mutually exclusive with the presence of oncogenes in CRC patients, exception of one patient expressing TMOD3-MAPK6 $(P=0.029)$, concurrent with previous reports wherein tumour samples harbouring fusion genes tended to have significantly fewer oncogene mutations. ${ }^{27,} 28$ However, cancer progression of the patient with TMOD3-MAPK6 may be affected by oncogene and tumour suppressor gene mutations involving $P O L E$, in addition to the fusion gene. ${ }^{4}$

We selected 11 fusion genes (APC- COMMD10, GTF3A-CDK8, LMNA-NTRK1, NAGLU-IKZF3, PTPRK-RSOP3, RASA1-LOC644100,
RNF121-FOLR2, STRN-ALK, TMOD3-MAPK6, TPM3-NTRK1, and TRIM24-BRAF) that were predicted as stronger oncogenes due to gene expression or function. Interestingly, we discovered fusion genes that comprised of tumour suppressor genes, APC and RASA1. Functional domain analysis showed truncation of $A P C$ in APC-COMMD10 (1-293 aa in 325 aa) and RASA1 in RASA1LOC644100 (1-179 aa in 253 aa). Truncated APC forms, especially those lacking the C-terminal, are commonly expressed in CRC, while wild type APC is expressed in normal tissue. ${ }^{29,} 30$ The betacatenin binding region (1020 aa-1638 aa) of APC is important for inhibiting Wnt signalling activation via cytosolic beta-catenin turnover. $^{29,}$, 31-33 Tighe et al. reported that truncated APC without beta-catenin-binding region initiated chromosomal instability, thereby exerting oncogenic effects. ${ }^{34}$ Therefore, truncated APC in $A P C-C O M M D 10$ could not have a tumour suppressor-like function because APC-COMMD10 does not have any functional domain. In addition, 120RasGAP coded by RASA1 converts active Ras-GTP to inactive Ras-GDP, which then inhibits RAS oncogene. ${ }^{35}$, ${ }^{36}$ At the molecular level, Src homology 2 (SH2), Src homology 3 $(\mathrm{SH} 3)$, pleckstrin homology $(\mathrm{PH})$, and calcium-dependent phospholipid-binding (C2) domains regulate cell proliferation, 

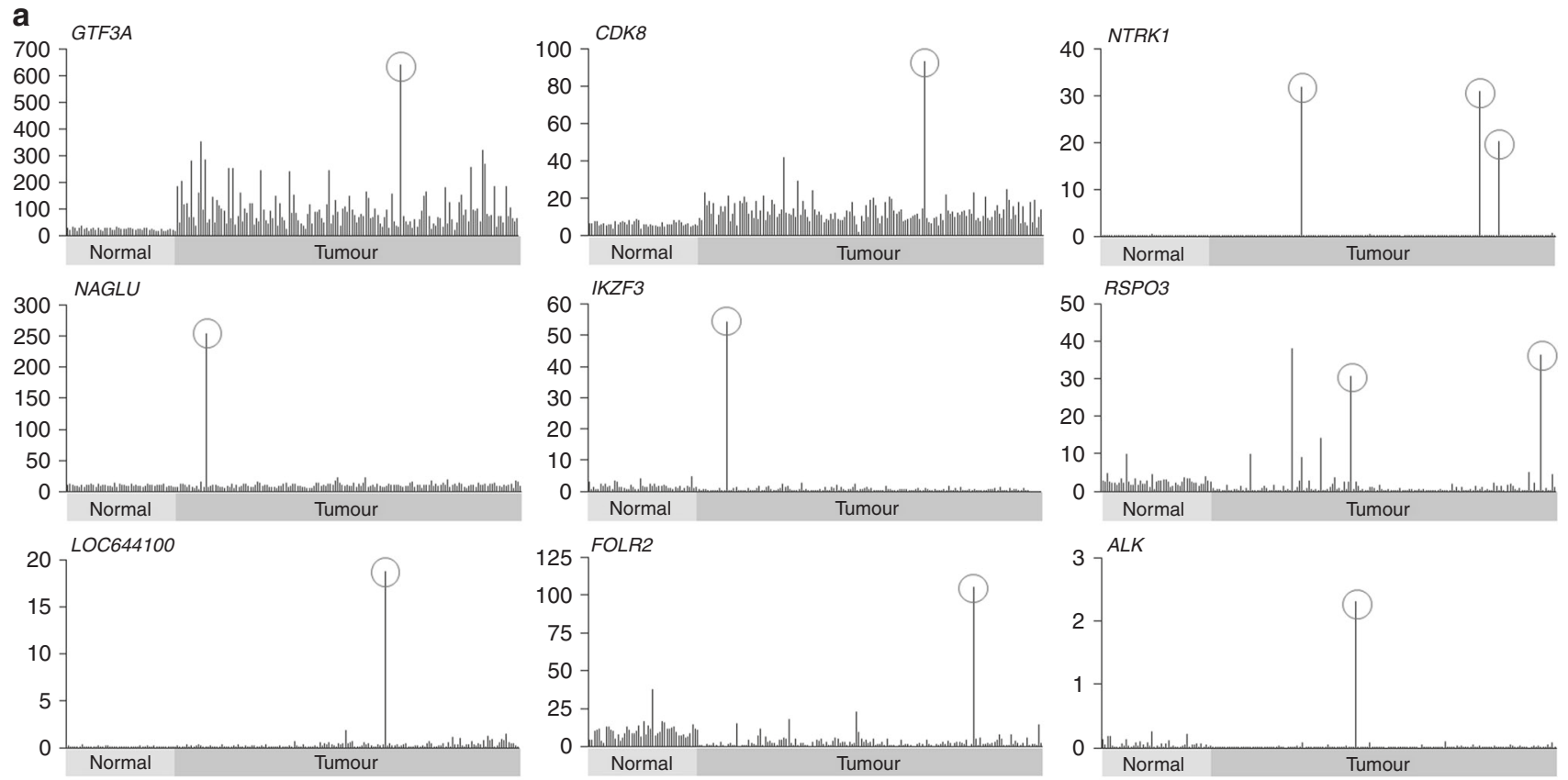

b
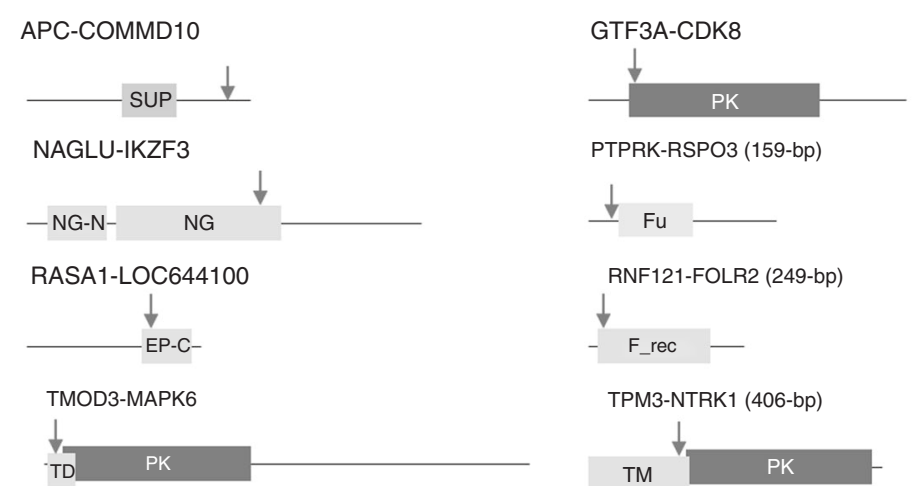

PTPRK-RSPO3 (159-bp)
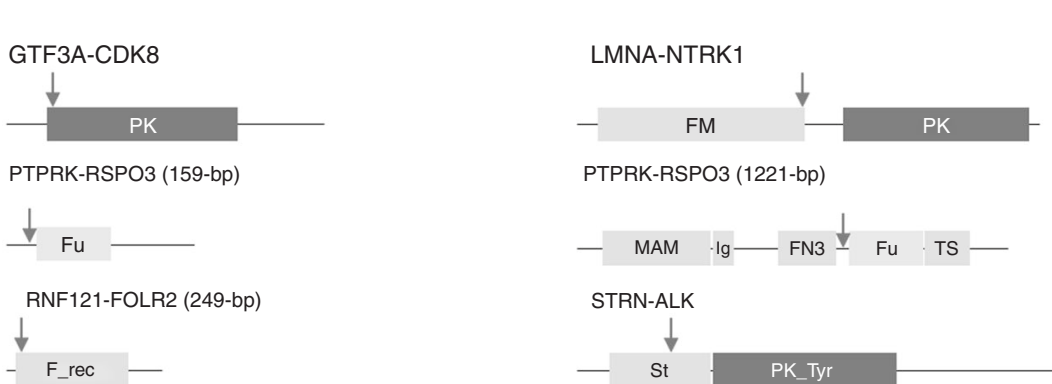

TPRK-RSPO3 (1221-bp)

TPM3-NTRK1 (712-bp)

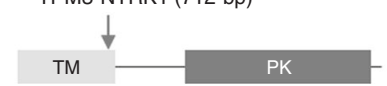

TPM3-NTRK1 (406-bp)
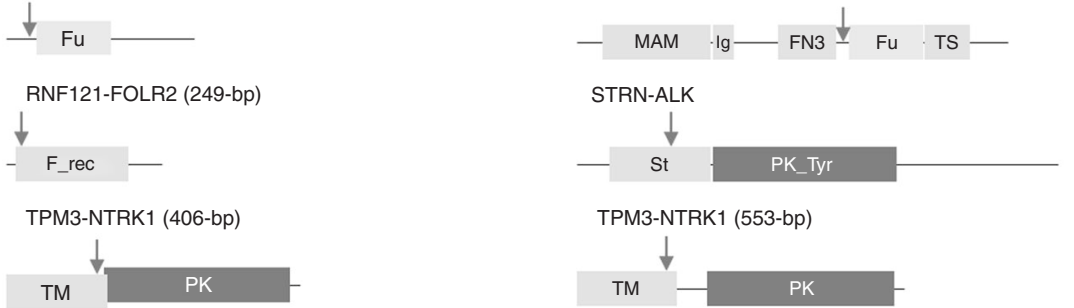

TPM3-NTRK1 (553-bp)

TRIM24-BRAF
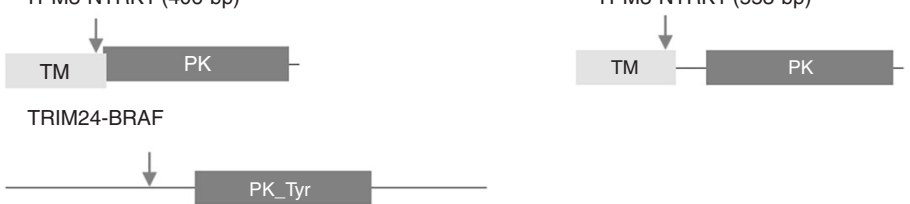

Fig. 3 Predictions of biological functions of fusion genes. a Gene expression of donor or acceptor genes. Only the gene expression data of patients with high expression of donor or acceptor genes were presented. Red circle depicts patient with each fusion gene. The X-axis represents 47 paired normal samples (sky blue) and 147 tumour samples (pink). The Y-axis represents fragments per kilobase million (FPKM). b Schematic protein structure of fusion genes. Red arrow indicates fusion site. Protein domain abbreviations are as follows: EP-C, ARF7 effector protein C-terminus; FM, filament; FN3, fibronectin type III; F_rec, folate receptor family; Fu, furin-like repeat and cysteine-rich; Ig, immunoglobulin I-set; MAM, meprin/A5/mu; NG, NAGLU tim-barrel; NG-N, NAGLU N-terminal; PK, protein kinase; PK Tyr, protein tyrosine kinase; St, striatin family; SUP, APC tumour suppressor protein; TD, tropomodulin; TM, tropomyosin; TS, thrombospondin type 1

migration, and apoptosis in accordance with their sub-binding partners. ${ }^{37-41}$ Tumour suppressor activity may be obliterated in RASA1-LOC644100, since the fused region of RASA1 does not involve all pivotal domains for inhibiting tumourigenesis. ${ }^{42}$ Therefore, its truncation in the fusion gene may promote tumourigenic mechanisms in CRC. These hypotheses were partially supported by the lack of somatic mutations of $A P C$ and RASA1 identified in our cohort of CRC samples that expressed APC and RASA1 fusion genes.

There are several discrepancies regarding the frequency and type of fusion genes in CRC between different studies. In our study, we identified 11 oncogenic fusion genes in $8.8 \%$ of our cohort. In comparison, Kloosterman et al. reported that only $2.5 \%$ of CRC expressed fusion genes, including ArfGAP with GTPase domain, ankyrin repeat, and PH domain 3 (AGAP3)-BRAF, TRIM24$B R A F$, discs large MAGUK scaffold protein 1 (DLG1)-BRAF, echinoderm microtubule associated protein like 4 (EML4)-NTRK3, ribosome binding protein 1 (RRBP1)-RET, USP9X-embryonic stem cell-expressed Ras (ERAS), and eukaryotic translation initiation factor 3 subunit E (EIF3E)-RSOP2 fusion genes. ${ }^{8}$ These discrepancies may be due to differences in sample collection and preparation and bioinformatics analysis pipeline. Furthermore, the functional significance of fusion genes in CRC varies between studies. Seshagiri et al. reported recurrent fusion genes involving R-spondin family members, RSOP2 and RSPO3, in $10 \%$ of colon cancers. ${ }^{7}$ However, Kloosterman et al., Shinmura et al., and our present study demonstrated that RSPO fusion genes were lowly expressed in colon cancers $\left(0.35 \%, 4 \%\right.$, and $1.36 \%$, respectively). ${ }^{8}$, 43 This may be due to sampling bias, bioinformatics tools, or selection bias, among other possible reasons. However, Kloosterman et al. suggested that the use of different bioinformatics tools between studies was not a major confounding factor. ${ }^{8}$ 
a
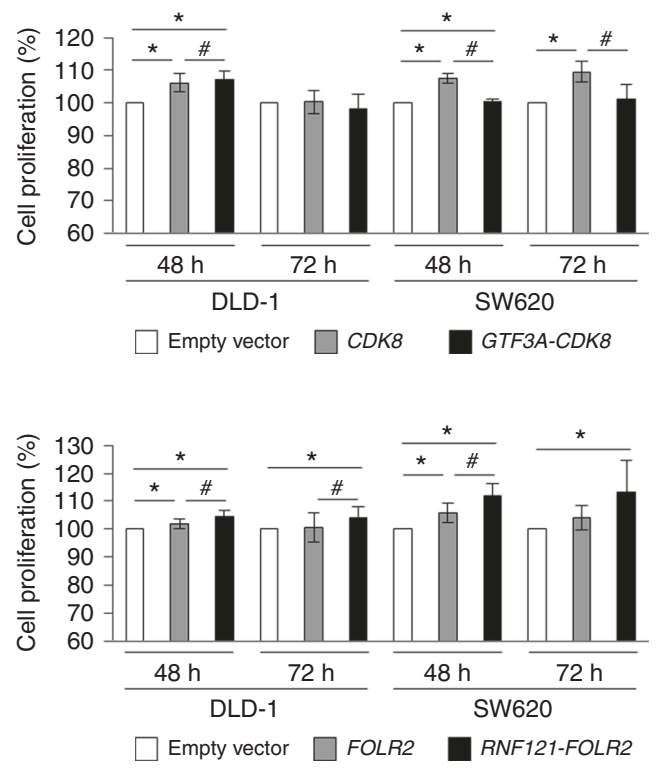

b

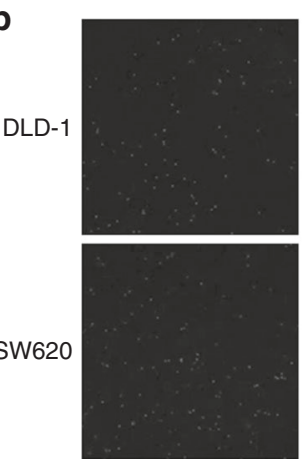

Empty vector
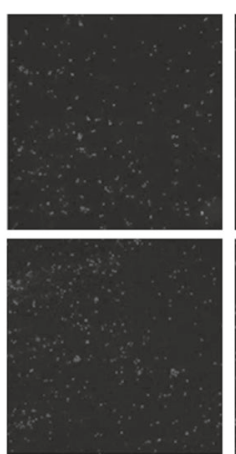

IKZF3

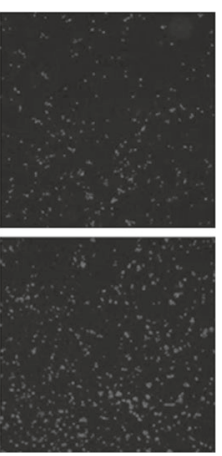

NAGLU-IKZF3
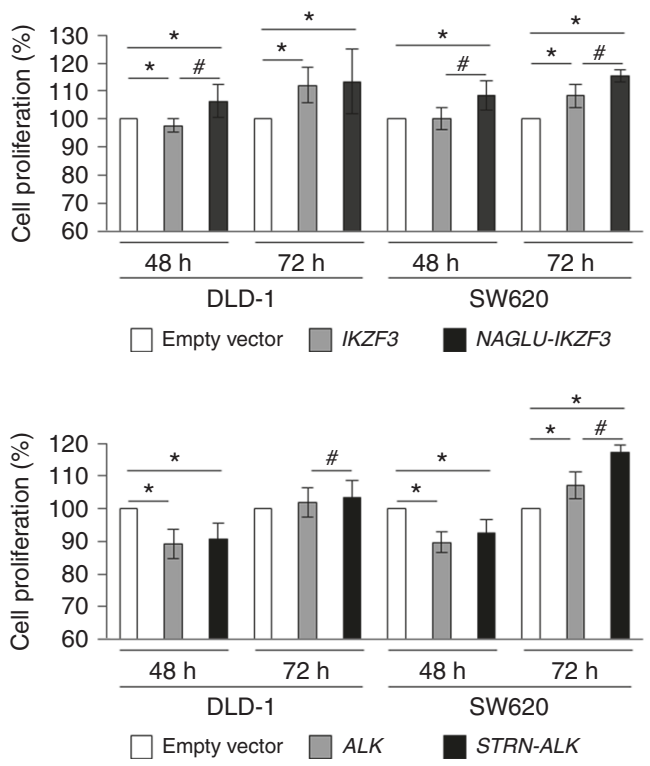

C

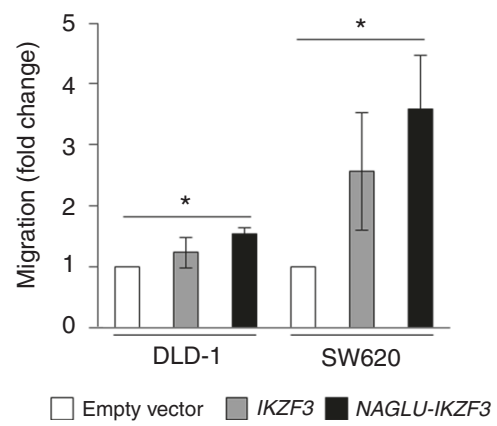

Fig. 4 Biological function test. a Cell proliferation in CRC cells. Each MTT assay was performed three times. Empty vector is pCMV6-myc-DDK, except RNF121-FOLR2, which used pcDNA3.1-V5-His B. ${ }^{*} P<0.05$ compared to empty vector, determined by the Student's $t$-test; ${ }^{\#} P<0.05$ compared to each acceptor gene expression vector, determined by the Student's $t$-test. $\mathbf{b}$ Cell migration assay of NAGLU-IKZF3 was performed three times. Harvested cells were seeded in transwell $48 \mathrm{~h}$ after transfection. ${ }^{*} P<0.05$ compared to empty vector, determined by the Student's $t$-test

Additional studies are necessary to investigate these discrepancies further.

In the present study, we performed functional analysis of four fusion genes, GTF3A-CDK8, NAGLU-IKZF3, RNF121-FOLR2, and STRN$A L K$. We validated the biological functions of the fusion genes in vitro. In this study, although CDK8 expressed intact protein kinase domain, overexpression of GTF3A-CDK8 did not have any effect on cell proliferation, contrary to our CDK8 overexpression data and a previous study in which CDK8 activated the Wnt/betacatenin signalling pathway in CRC. ${ }^{44}$ The CDK8 kinase module, comprised of mediator 12 , mediator 13 , cyclin $C$, and CDK8, binds beta-catenin, which promotes the transcription of oncogenes. ${ }^{45}$ The aB helix ( 3 aa-12 aa) at the N-terminal of CDK8 is important for recognition of cyclin $C_{1}^{46}$ and the $C D K 8 /$ cyclin $C$ complex recruits the remaining components of the CDK8 kinase module, mediator 12 and $13 .{ }^{47}$ In CRC overexpressing GTF3A-CDK8, the CDK8 module may not properly form due to the absence of the region that interacts with cyclin $C$ in the fusion gene. Thus, we suggest that the $\mathrm{N}$-terminal of CDK8 may play a pivotal role in oncogenesis in CRC.

Overexpression of NAGUL-IKZF3 significantly increased cell growth and migration, compared to cells overexpressing only IKZF3. IKZF3 expression was previously demonstrated to mediate cancer metastasis by promoting anchorage independence, ${ }^{48}$ and cell proliferation of a breast cancer cell line possessing VAMP-associated protein $B$ and C (VAPB)-IKZF3 was suppressed when IKZF3 was knocked down. ${ }^{49} \mathrm{We}$ demonstrated that NAGUL-IKZF3 expression may influence both tumourigenesis and metastasis in CRC.

CRC cells overexpressing RNF121-FOLR2 exhibited increased cell proliferation. Folate receptor beta, coded by FOLR2, was reported to localise to activated macrophages that amass in tumours and at inflammation sites. ${ }^{50-52}$ Folate receptor beta was expressed in tumour-associated macrophages (TAMs) that caused immune tolerance, enhancing angiogenesis, increasing tumour cell migratory and invasive abilities, and reducing cell apoptosis and sensitivity to anticancer drugs. ${ }^{53-55}$ It is unclear whether FOLR2 expression in tumour cells recruits TAMs or TAMs highly express FOLR2. Regardless, immunotoxins targeting folate receptor beta reduced tumour growth in glioma xenograft models. ${ }^{50}$ Hence, the presence of RNF121-FOLR2 may be a potential prognostic marker for folate-mediated, anti-inflammatory drugs.

Overexpression of STRN-ALK increased CRC cell proliferation. Cells expressing STRN-ALK increased thyroid-stimulating, hormone-independent cell proliferation and developed tumours in nude mice. ${ }^{16}$ The ALK inhibitor, crizotinib, was approved by the US Food and Drug Administration as a chemotherapeutic treatment for lung cancer patients positive for EML4-ALK. ${ }^{56}$ Although the function of STRN-ALK related with CRC was not 
studied in vitro, Yakirevich et al. conducted a clinical trial of the ALK inhibitor, ceritinib, which was 20-fold more effective than crizotinib, on a cohort that included a CRC patient positive for STRN-ALK in a pre-clinical setting. ${ }^{17}$ Ceritinib treatment decreased tumour size of the patient with STRN-ALK, but resistance to ceritinib developed after 9 months. ${ }^{17}$ Therefore, we also suggest that ALK inhibitors may be provided to patients positive for STRN$A L K$ as an initial drug.

In conclusion, we comprehensively analysed fusion genes via various fusion algorithms, clinicopathological information, and SNV data in CRC patients, identified the significant fusion genes, and investigated their function. We demonstrated the oncogenic capacity of several fusion genes detected in this study through analysis of SNVs, gene expression, and gene function, and we determined that NAGLU-IKZF3, RNF121-FOLR2, and STRN-ALK had tumourigenic effects in CRC. Therefore, these fusion genes may be good candidates as molecular targets for the development of cancer therapy in precision medicine.

\section{ACKNOWLEDGEMENTS}

The biospecimens for this study were provided by the Pusan National University Hospital and Chonnam National University Hwasun Hospital or by the members of the National Biobank of Korea, which is supported by the Ministry of Health, Welfare, and Family Affairs.

\section{AUTHORS CONTRIBUTIONS}

Y.C. designed the study, analysed the data, performed the experiments, and wrote the manuscript. D.Y.P. obtained funding, designed the study, wrote and reviewed the manuscript. C.H.K. designed the study, analysed the data, and reviewed the manuscript. S.J.L. performed the experiments. J.P. and J.Y.S. produced the NGS data.

\section{ADDITIONAL INFORMATION}

Supplementary information is available for this paper at https://doi.org/10.1038/ s41416-018-0153-3.

Ethics approval and consent to participate: This study was conducted according to the Good Clinical Practice guidelines and the Declaration of Helsinki. The protocol was approved by the institutional review board or ethics committee at Pusan National University Hospital and Chonnam National University Hwasun Hospital, and written informed consent was obtained for all patients.

Funding: This work was supported by Busan Cancer Center Research grant (2018) by Pusan National University Hospital.

Competing interests: The authors declare no competing interests.

Note: This work is published under the standard license to publish agreement. After 12 months the work will become freely available and the license terms will switch to a Creative Commons Attribution 4.0 International (CC BY 4.0).

\section{REFERENCES}

1. Siegel, R. L., Miller, K. D. \& Jemal, A. Cancer statistics, 2015. CA Cancer J. Clin. 65 5-29 (2015).

2. Sobrero, A. F. et al. EPIC: phase III trial of cetuximab plus irinotecan after fluoropyrimidine and oxaliplatin failure in patients with metastatic colorectal cancer. $J$. Clin. Oncol. 26, 2311-2319 (2008).

3. Giantonio, B. J. et al. Bevacizumab in combination with oxaliplatin, fluorouracil, and leucovorin (FOLFOX4) for previously treated metastatic colorectal cancer: results from the Eastern Cooperative Oncology Group Study E3200. J. Clin. Oncol. 25, 1539-1544 (2007)

4. Cancer Genome Atlas Network. Comprehensive molecular characterization of human colon and rectal cancer. Nature 487, 330-337 (2012).

5. Goldman, J. M. \& Melo, J. V. Chronic myeloid leukemia - advances in biology and new approaches to treatment. N. Engl. J. Med. 349, 1451-1464 (2003)

6. Forde, P. M. \& Rudin, C. M. Crizotinib in the treatment of non-small-cell lung cancer. Expert Opin. Pharmacother. 13, 1195-1201 (2012).
7. Seshagiri, S. et al. Recurrent R-spondin fusions in colon cancer. Nature 488 , 660-664 (2012).

8. Kloosterman, W. P. et al. A systematic analysis of oncogenic gene fusions in primary colon cancer. Cancer Res. 77, 3814-3822 (2017).

9. Park, D. Y. et al. NTRK1 fusions for the therapeutic intervention of Korean patients with colon cancer. Oncotarget 7, 8399-8412 (2016).

10. Ju, Y. S. et al. Extensive genomic and transcriptional diversity identified through massively parallel DNA and RNA sequencing of eighteen Korean individuals. Nat. Genet. 43, 745-752 (2011).

11. McPherson, A. et al. deFuse: an algorithm for gene fusion discovery in tumour RNA-Seq data. PLoS Comput. Biol. 7, e1001138 (2011).

12. $\mathrm{Ge}, \mathrm{H}$. et al. FusionMap: detecting fusion genes from next-generation sequencing data at base-pair resolution. Bioinformatics 27, 1922-1928 (2011).

13. International HapMap 3 Consortium, Altshuler, D. M. et al. Integrating common and rare genetic variation in diverse human populations. Nature 467, 52-58 (2010).

14. Jeong, J. Y. et al. One-step sequence-and ligation-independent cloning as a rapid and versatile cloning method for functional genomics studies. Appl. Environ. Microbiol. 78, 5440-5443 (2012).

15. Majewski, I. J. et al. Identification of recurrent FGFR3 fusion genes in lung cancer through kinome-centred RNA sequencing. J. Pathol. 230, 270-276 (2013).

16. Kelly, L. M. et al. Identification of the transforming STRN-ALK fusion as a potential therapeutic target in the aggressive forms of thyroid cancer. Proc. Natl Acad. Sci. USA 111, 4233-4238 (2014).

17. Yakirevich, E. et al. Oncogenic ALK fusion in rare and aggressive subtype of colorectal adenocarcinoma as a potential therapeutic target. Clin. Cancer Res. 22, 3831-3840 (2016).

18. Klijn, C. et al. A comprehensive transcriptional portrait of human cancer cell lines. Nat. Biotechnol. 33, 306-312 (2015).

19. Cancer Genome Atlas Research Network. Comprehensive molecular characterization of gastric adenocarcinoma. Nature 513, 202-209 (2014).

20. $\mathrm{Hu}, \mathrm{X}$. et al. TumorFusions: an integrative resource for reporting cancer-associated transcript fusions in 33 tumor types. Nucleic Acids Res. 46, 1144-1149 (2018).

21. Nome, T. et al. Common fusion transcripts identified in colorectal cancer cell lines by high-throughput RNA sequencing. Transl. Oncol. 6, 546-553 (2013).

22. Kalvala, A. et al. Rad51C-ATXN7 fusion gene expression in colorectal tumors. Mol. Cancer 15, 47 (2016).

23. Tognon, $C$. et al. Expression of the ETV6-NTRK3 gene fusion as a primary event in human secretory breast carcinoma. Cancer Cell. 2, 367-376 (2002).

24. Inamura, K. et al. EML4-ALK lung cancers are characterized by rare other mutations, a TTF-1 cell lineage, an acinar histology, and young onset. Mod. Pathol. 22, 508-515 (2009).

25. Persson, M. et al. Recurrent fusion of MYB and NFIB transcription factor genes in carcinomas of the breast and head and neck. Proc. Natl. Acad. Sci. USA 106, 18740-18744 (2009).

26. Weigelt, B., Geyer, F. C. \& Reis-Filho, J. S. Histological types of breast cancer: how special are they? Mol. Oncol. 4, 192-208 (2010).

27. Cheng, L. et al. Molecular pathology of lung cancer: key to personalized medicine. Mod. Pathol. 25, 347-369 (2012).

28. Yoshihara, K. et al. The landscape and therapeutic relevance of cancer-associated transcript fusions. Oncogene 34, 4845-4854 (2015).

29. Schneikert, J., Grohmann, A. \& Behrens, J. Truncated APC regulates the transcriptional activity of beta-catenin in a cell cycle dependent manner. Hum. Mol. Genet. 16, 199-209 (2007).

30. Barua, D. \& Hlavacek, W. S. Modeling the effect of APC truncation on destruction complex function in colorectal cancer cells. PLoS Comput. Biol. 9, e1003217 (2013).

31. Voloshanenko, O. et al. Wnt secretion is required to maintain high levels of Wnt activity in colon cancer cells. Nat. Commun. 4, 2610 (2013).

32. Su, L. K., Vogelstein, B. \& Kinzler, K. W. Association of the APC tumor suppressor protein with catenins. Science 262, 1734-1737 (1993).

33. Rubinfeld, B. et al. Association of the APC gene product with beta-catenin. Science 262, 1731-1734 (1993).

34. Tighe, A., Johnson, V. L. \& Taylor, S. S. Truncating APC mutations have dominant effects on proliferation, spindle checkpoint control, survival and chromosome stability. J. Cell Sci. 117, 6339-6353 (2004).

35. Orme, C. M., Boyden, L. M., Choate, K. A., Antaya, R. J. \& King, B. A. Capillary malformation-arteriovenous malformation syndrome: review of the literature, proposed diagnostic criteria, and recommendations for management. Pediatr. Dermatol. 30, 409-415 (2013).

36. Kulkarni, S. V., Gish, G., van der Geer, P., Henkemeyer, M. \& Pawson, T. Role of p120 Ras-GAP in directed cell movement. J. Cell Biol. 149, 457-470 (2000).

37. Trahey, M. et al. Molecular cloning of two types of GAP complementary DNA from human placenta. Science 242, 1697-1700 (1988). 
38. Clark, J. D. et al. A novel arachidonic acid-selective cytosolic PLA2 contains a Ca(2 + -)dependent translocation domain with homology to PKC and GAP. Cell 65, 1043-1051 (1991).

39. Moran, M. F. et al. Src homology region 2 domains direct protein-protein interactions in signal transduction. Proc. Natl Acad. Sci. USA 87, 8622-8626 (1990).

40. Musacchio, A., Gibson, T., Rice, P., Thompson, J. \& Saraste, M. The PH domain: a common piece in the structural patchwork of signalling proteins. Trends Biochem. Sci. 18, 343-348 (1993).

41. Gawler, D. J., Zhang, L. J., Reedijk, M., Tung, P. S. \& Moran, M. F. CaLB: A 43 amino acid calcium-dependent membrane/phospholipid binding domain in p120 Ras GTPase-activating protein. Oncogene 10, 817-825 (1995).

42. Pamonsinlapatham, P. et al. p120-Ras GTPase activating protein (RasGAP): a multi-interacting protein in downstream signaling. Biochimie 91, 320-328 (2009).

43. Shinmura, K. et al. RSPO fusion transcripts in colorectal cancer in Japanese population. Mol. Biol. Rep. 41, 5375-5384 (2014).

44. Firestein, R. et al. CDK8 is a colorectal cancer oncogene that regulates $\beta$-catenin activity. Nature 455, 547-551 (2008).

45. Galbraith, M. D., Donner, A. J. \& Espinosa, J. M. CDK8: a positive regulator of transcription. Transcription 1, 4-12 (2010),

46. Schneider, E. V. et al. The structure of CDK8/CycC implicates specificity in the $\mathrm{CDK} /$ cyclin family and reveals interaction with a deep pocket binder. J. Mol. Biol. 412, 251-266 (2011)

47. Knuesel, M. T., Meyer, K. D., Donner, A. J., Espinosa, J. M. \& Taatjes, D. J. The human CDK8 subcomplex is a histone kinase that requires Med12 for activity and can function independently of mediator. Mol. Cell. Biol. 29, 650-661 (2009).
48. $\mathrm{Li}, \mathrm{X}$. et al. Aiolos promotes anchorage independence by silencing p66 Shc transcription in cancer cells. Cancer Cell. 25, 575-589 (2014).

49. Billot, K. et al. Differential aiolos expression in human hematopoietic subpopulations. Leuk. Res. 34, 289-293 (2010).

50. Nagai, T. et al. Targeting tumor-associated macrophages in an experimental glioma model with a recombinant immunotoxin to folate receptor beta. Cancer Immunol. Immunother. 58, 1577-1586 (2009).

51. van der Heijden, J. W. et al. Folate receptor beta as a potential delivery route for novel folate antagonists to macrophages in the synovial tissue of rheumatoid arthritis patients. Arthritis Rheum. 60, 12-21 (2009).

52. Varghese, B., Haase, N. \& Low, P. S. Depletion of folate-receptor-positive macrophages leads to alleviation of symptoms and prolonged survival in two murine models of systemic lupus erythematosus. Mol. Pharm. 4, 679-685 (2007).

53. Puig-Kröger, A. et al. Folate receptor $\beta$ is expressed by tumor-associated macrophages and constitutes a marker for $\mathrm{M} 2$ anti-inflammatory/regulatory macrophages. Cancer Res. 69, 9395-9403 (2009).

54. Shen, J. et al. Assessment of folate receptor- $\beta$ expression in human neoplastic tissues. Oncotarget 6, 14700-14709 (2015).

55. O'Shannessy, D. J., Somers, E. B., Wang, L. C., Wang, H. \& Hsu, R. Expression of folate receptors alpha and beta in normal and cancerous gynecologic tissues: correlation of expression of the beta isoform with macrophage markers. J. Ovarian Res. 8, 29 (2015).

56. Kwak, E. L. et al. Anaplastic lymphoma kinase inhibition in non-small-cell lung cancer. N. Engl. J. Med. 363, 1693-1703 (2010). 